\title{
Plantas utilizadas en cercos vivos de ciudades patagónicas: aportes de la etnobotánica para la conservación
}

\author{
Adriana E. Rovere ${ }^{1, \varpi}$; Soledad Molares ${ }^{2} \&$ Ana H. Ladio 3 \\ 1. Consejo Nacional de Investigaciones Científicas y Técnicas (CONICET), Universidad Nacional del Comahue, Bariloche, \\ Argentina. 2.Consejo Nacional de Investigaciones Científicas y Técnicas (CONICET), Universidad Nacional de la Patagonia San \\ Juan Bosco, Esquel, Argentina. 3. Instituto de Investigaciones en Biodiversidad y Medioambiente (INIBIOMA), Universidad \\ Nacional del Comahue, CONICET Consejo Nacional de Investigaciones Científicas y Técnicas, Bariloche, Argentina.
}

\begin{abstract}
Resumen. Los cercos vivos son parte significativa del paisaje de las ciudades de la Patagonia y pueden ser muy importantes no sólo como reservorios de biodiversidad sino también como posibles fuentes de invasiones. En este trabajo se estimó la riqueza de especies, sus familias botánicas y el origen biogeográfico de las plantas seleccionadas como cercos vivos en ocho ciudades turísticas de la Patagonia. Si se considera la influencia que los colonos europeos han tenido sobre el desarrollo cultural y paisajístico de la región, es esperable una proporción elevada de recursos de origen Holártico. Se identificaron las especies en 100 casas elegidas al azar por localidad (San Martín de los Andes, Villa la Angostura, Dina Huapi, El Bolsón, Lago Puelo, Esquel y Trevelin) y 300 para Bariloche dado el mayor tamaño de la ciudad. La riqueza total correspondió a 137 especies, y las más comunes fueron: Cytisus scoparius (L.) Link (9\%), Rosa sp. (8\%), Pseudotsuga menziesii (Mirb.) Franco (7\%), Cupressus macrocarpa Hartw. ex Gordon (5\%) y Ligustrum sinense Lour. (3\%). De las 48 familias botánicas representadas, las más frecuentes fueron: Rosaceae, Fabaceae, Cupressaceae y Pinaceae (67\% del total del registro). Las especies provienen de la región Holártica (72\%), de la Antártica (23\%), Paleotropical $(19 \%)$, Neotropical (5\%) y Australiana (2\%). Se concluye que los cercos estudiados muestran en términos de riqueza de especies, similitudes con las principales especies ornamentales a nivel global. Sin embargo, la presencia de elementos antárticos (32 especies) indicaría la incorporación de especies nativas de los bosques andino-patagónicos en los criterios locales, por ende, con un valioso papel a nivel de la conservación. Por otra parte, el relevamiento muestra que ciertas especies pueden representar riesgos de invasiones biológicas (e.g., Sorbus aucuparia L., Crataegus monogyna Jacq., Ulex europaeus L.), por lo que se propone la difusión de sus efectos negativos en la población.
\end{abstract}

[Palabras clave: exóticas, invasiones, leñosas, nativas, ornamentales, restauración]

Aвstract. Plants used for hedging in Patagonian cities: an ethnobotanical contribution to conservation: Hedges constitute a significant part of Patagonian city landscapes, and can be very important not only as reservoirs of biodiversity, but also as possible sources of invasion. In this work, carried out in eight Patagonian tourist cities, species richness was estimated and the botanical families and biogeographical origin of the plants chosen for hedges were recorded. Considering the influence of European colonists on cultural and landscaping development in the region, one would expect a high proportion of resources to be of Holarctic origin. Species were identified in 100 homes chosen at random in each locality (San Martín de los Andes, Villa la Angostura, Dina Huapi, El Bolsón, Lago Puelo, Esquel and Trevelin) and in 300 homes in Bariloche, due to its greater size. Total plant richness was 137 species, the most common being Cytisus scoparius (L.) Link (9\%), Rosa sp. (8\%), Pseudotsuga menziesii (Mirb.) Franco (7\%), Cupressus macrocarpa Hartw. ex Gordon (5\%) and Ligustrum sinense Lour. (3\%). Of the 48 botanical families represented, the most frequent were Rosaceae, Fabaceae, Cupressaceae and Pinaceae (67\% of the total registered). Species come from the following regions: Holarctic (72\%), Antarctic $(23 \%)$, Paleotropical (19\%), Neotropical (5\%) and Australian (2\%). We conclude that the hedges studied, in terms of species richness, display similarities with the main ornamental species at a global level. However, the presence of Antarctic elements (32 species) indicates the incorporation of species native to Andean-Patagonian forests, and therefore an important role is being played here in terms of conservation. In addition, our survey shows that certain species may present a risk of biological invasion (e.g., Sorbus aucuparia L., Crataegus monogyna Jacq., Ulex europaeus L.) and we therefore propose that the population should be warned about the negative effects of these species.

[Keywords: exotic, invasions, native, ornamental, restoration, woody plants]

\section{INTRODUCCIÓN}

El paisaje urbano que es creado y recreado por los habitantes de las ciudades tiene un rol significativo por las numerosas funciones ecosistémicas y socioculturales que sustenta
(McKinney 2006; Rodríguez-Laredo 2008). Las floras urbanas garantizan la interacción de los seres humanos con los elementos del mundo natural y propician vínculos materiales y espirituales (Siviero et al. 2011). Sin embargo, 
hasta el momento han sido poco estudiadas (Méndez 2005) y/o incluidas como escenarios de relevancia en las políticas de conservación de Argentina.

El papel de la flora urbana en los ecosistemas presenta aspectos positivos y negativos. Por un lado, ha sido considerada por su papel como reservorio de biodiversidad y vida silvestre (Ladio \& Damascos 2000; Rudd et al. 2002; McKinney 2006; Nagendra \& Gopal 2011) y como corredor biológico facilitando la conectividad con unidades de conservación cercanas (Rudd et al. 2002). Estos espacios vegetados mejoran la calidad del aire al reducir los contaminantes atmosféricos, actúan de reguladores de variaciones climáticas, disminuyen el nivel de ruido y regulan el flujo de agua, entre otras funciones (Nagendra \& Gopal 2011). Las floras de las ciudades pueden ser vistas como escenarios en donde se enriquece la diversidad específica vertical y horizontal del paisaje, se favorece el mantenimiento de ciclos ecosistémicos generales y se genera una menor presión de uso de recursos vegetales de las áreas silvestres circundantes (Ospina-Ante 2003). A su vez, ofrecen un espacio de esparcimiento y de educación, con amplios beneficios terapéutico-psicológico-emocionales en la población (Rodríguez-Laredo 2008; Linhares de Souza et al. 2012). Por otro lado, la flora urbana también puede ser fuente de propágulos de especies invasoras, lo que genera perjuicios ambientales y económicos graves (Rapoport 1988; Ladio \& Damascos 2000). Se ha documentado que en numerosas localidades balnearias del norte de la costa atlántica argentina la expansión de los núcleos urbanos favoreció a las especies introducidas desplazando la flora nativa, lo cual resalta la importancia de estos espacios durante la planificación urbana (Faggi \& Dadon 2010). Así, el paisaje urbano puede ser visto como parte de un proceso de homogeneización y/o conversión de la vegetación que impulsa la pérdida de biodiversidad nativa del lugar (McKinney 2006). Existen patrones globales de selección de plantas ornamentales que orientan a las sociedades a que sus floras sean muy similares (Rapoport 1988). Por lo tanto, es necesaria una visión integradora que contemple la funcionalidad, presente y/ o futura, de estos espacios como unidades de conservación de custodia particular o privada y que pueda ser articulada con otras políticas de conservación en áreas públicas.
Las floras urbanas incluyen, por un lado, a los parques y el arbolado urbano a cargo de las autoridades locales, y por el otro, a los jardines, quintas, y/o cercos vivos particulares que dependen directamente de la selección sus habitantes. En particular, los cercos vivos son una parte importante de la vivienda, cuya principal función es delimitar a la propiedad, además de ser barrera visual, sonora y física, atenuar el viento y el polvo, y actuar como filtros ambientales (Rodríguez-Laredo 2008; Nascimento et al. 2009). Estos límites vivos son interesantes porque los cercos suelen ser las primeras intervenciones del paisaje que los vecinos realizan en sus casas. En este sentido, este estudio indaga cuáles son las plantas que los habitantes patagónicos seleccionan para delimitar su hogar (Vogl et al. 2004).

En términos generales, los estudios etnobotánicos dan cuenta que los cercos están compuestos por especies leñosas dispuestas en forma lineal, que cumplen la función de límite y protección (Ayuk 1997; Teruya Eichemberg et al. 2009). La mayor altura, la presencia de espinas y la textura densa del follaje de las plantas parecen ser rasgos distintivos (Rodríguez-Laredo 2008). Las especies elegidas como cerco a menudo poseen otros atributos funcionales que influyen en los criterios de su selección, como su valor estético-ornamental (Teruya Eichemberg et al. 2009; Cardoso \& Ladio 2011), su valor alimenticio y/o medicinal adicional (Nascimento et al. 2009; Teruya Eichemberg et al. 2009), sirven como forrajeras, brindan materiales para construcción, sirven como combustible (Cardoso \& Ladio 2011) y controlan el movimiento de animales domésticos y/o personas (Ayuk 1997; Ospina-Ante 2003; Rodríguez Laredo 2008; Nascimento et al. 2009). Los cercos constituyen estructuras muy comunes en áreas rurales y urbanas y representan un elemento importante en el paisaje de regiones tanto tropicales como templadas. Diferentes estudios en varias regiones de América han mostrado que existen componentes de la flora nativa formando parte de cercos y jardines; los ejemplares de distintas especies nativas son tolerados y dejados en pie aunque el espacio sea destinado para el cultivo de otras plantas (Duque-Brasil et al. 2011). Además, estas comunidades peridomésticas están sujetas a prácticas de manejo adicional por parte de los habitantes (e.g., por medio del raleo de malezas, protección contra heladas, pestes y/o competidores), así como también son enriquecidas por el traslado de propágulos 
sexuales y asexuales de plantas nativas provenientes de áreas silvestres (Eyssartier et al. 2011; Parra et al. 2012).

Las ciudades del noroeste de la Patagonia se caracterizan por un desarrollo histórico que ha estado muy influenciado por la creación de los Parques Nacionales del sur del país y por el fuerte impulso, desde la creación del gobierno argentino, de un poblamiento a base de inmigración principalmente europea (Valverde et al. 2011). Las ideas conservacionistas prevalentes, ligadas a la exclusión de los seres humanos de áreas protegidas (Sedrez Dos Reis \& Ladio 2012), en conjunción con objetivos geopolíticos (Bandieri 2005), primaron la creación de asentamientos a lo largo de la cordillera de los Andes e implicaron la exclusión de los pueblos originarios de su territorio (Bandieri 2005). Luego, desde la creación de los primeros parques nacionales en el sur, del parque nacional Nahuel Huapi en 1934 y después de los parques nacionales Lanín, Los Alerces, Lago Puelo, Los Glaciares y Perito Moreno, estos asentamientos humanos, que poco a poco se trasformaron en urbes, quedaron colindantes, próximos o incluso dentro de dichas unidades de conservación (Mermoz et al. 2009).

El establecimiento y la expansión de los centros urbanos en la Patagonia han estado acompañados de la modificación de la vegetación natural y su posterior reemplazo por especies exóticas, muchas de ellas consideradas invasoras (Ladio \& Damascos 2000; Rovere \& Molares 2012). Está muy bien documentado que el uso de especies exóticas ornamentales conlleva el riesgo de $\mathrm{su}$ asilvestramiento (naturalización) o, en el peor de los casos, de invasión biológica (Rapoport 1988). Las invasiones de plantas exóticas han sido extensamente estudiadas en la región (Simberloff et al. 2002; Sarasola et al. 2006), pero con el crecimiento de las ciudades, el papel de los habitantes urbanos en la introducción de nuevas especies ha sido poco evaluado (Ladio \& Molares 2010).

En Patagonia no hay estudios sobre la flora urbana y los cercos. Algunos trabajos refieren que los paisajes urbanos fueron modelados por la influencia y el aporte de los colonos europeos. Entre otras prácticas culturales, los colonos intentaron reproducir y evocar el paisaje vegetal de su patria natal al priorizar el uso de distintas especies exóticas conocidas en el viejo mundo (Haene \& Aparicio 2007). En el presente, a pesar de que existen ejemplos de trabajos sobre el arbolado urbano (Álvarez et al. 2009; Molina 2009; Puntieri \& Grosfeld 2009), no se han realizado relevamientos a nivel regional que permitan comprender cuáles son las especies preferidas por las personas ni los criterios predominantes. Por ello, este trabajo constituye el primer estudio que analiza la diversidad de especies leñosas, nativas y exóticas, utilizadas en la construcción de cercos vivos de la región andino nor-patagónica y su papel en términos de conservación. Nuestra aproximación fue indagar directamente a los cercos domiciliarios, tomando a estos espacios como el resultado tangible de la expresión de los criterios, percepciones y motivaciones de los pobladores acerca de su ámbito peridoméstico.

Los objetivos específicos de este trabajo son: 1) analizar y caracterizar al conjunto de especies que son utilizadas como cerco, sus familias botánicas y distinguir aquellas con mayor consenso de uso a nivel regional, 2) cuantificar las formas de vida de las principales especies utilizadas, 3) analizar el origen de las especies registradas, en relación al número de especies nativas y exóticas como así también a su origen biogeográfico, 4) diagnosticar a las especies exóticas que pueden implicar peligros en términos de invasiones. La hipótesis general de trabajo sostiene que al considerar las influencias que los colonos europeos tuvieron sobre el desarrollo cultural y paisajístico de la región se espera una alta proporción de especies exóticas de origen Holártico en los cercos patagónicos. Por otra parte se espera que existan representantes de la flora nativa que hayan sido tolerados y/o cultivados intencionalmente por los habitantes en sus peri-domicilios.

\section{MÉTOdos}

\section{Área de estudio}

El trabajo se realizó en ocho localidades de la Patagonia andina (Provincias de Neuquén, Río Negro y Chubut) colindantes a Parques Nacionales. Las ciudades de norte a sur fueron: San Martín de los Andes, Villa la Angostura, Dina Huapi, San Carlos de Bariloche, El Bolsón, Lago Puelo, Esquel y Trevelin. El clima de la región es templado-frio y húmedo, con un régimen mediterráneo de precipitaciones (lluvias y nevadas concentradas en invierno).

La ciudad de San Martín de los Andes (4009' S y $71^{\circ} 21^{\prime}$ O) está ubicada en un paisaje de bosque 
andino-patagónico y se encuentra lindante al área protegida parque nacional Lanín. Posee una población de 23519 habitantes. La ciudad de Villa la Angostura $\left(40^{\circ} 45^{\prime} \mathrm{S}\right.$ y $\left.71^{\circ} 38^{\prime} \mathrm{O}\right)$ también está situada dentro del bosque andino-patagónico neuquino, se ubica en el sector norte del parque nacional Nahuel Huapi y también constituye el punto de ingreso al parque nacional los Arrayanes. Posee una población de 7526 habitantes. La localidad de Dina Huapi $\left(41^{\circ} 07^{\prime} \mathrm{S}\right.$ y $\left.70^{\circ} 40^{\prime} \mathrm{O}\right)$ se halla a 15 kilómetros de la ciudad de San Carlos de Bariloche, está emplazada en un paisaje de ecotono bosque-estepa patagónica y colinda con el parque Nahuel Huapi. Tiene una población de 2243 habitantes. La ciudad de San Carlos de Bariloche $\left(41^{\circ} 08^{\prime} S\right.$ y $\left.71^{\circ} 18^{\prime} \mathrm{O}\right)$ se ubica en el centro del parque nacional Nahuel Huapi. Su población es de 133500 habitantes. La ciudad de El Bolsón (415ㅇ' S y 71³2' O) se ubica a 30 km de la ciudad de Lago Puelo, próxima al Parque Lago Puelo. El Bolsón posee una población de 15537 habitantes. Lago Puelo (41⒌ $58^{\prime} \mathrm{S}$ y $\left.71^{\circ} 32^{\prime} \mathrm{O}\right)$ es una localidad que se ubica al noroeste de la provincia del Chubut. Colinda con el Parque Lago Puelo, que se caracteriza por resguardar especies de los bosques andino-patagónicos y elementos característicos de la selva valdiviana. La localidad Lago Puelo posee una población de 4046 habitantes. La ciudad de Esquel ( $42^{\circ} 54^{\prime} S$ y $\left.71^{\circ} 18^{\prime} \mathrm{O}\right)$ es adyacente al parque nacional Los Alerces y está a $24 \mathrm{~km}$ de la ciudad de Trevelin. Su población es de 39848 habitantes. La ciudad de Trevelin ( $43^{\circ} 05^{\prime} \mathrm{S}$ y $\left.71^{\circ} 17^{\prime} \mathrm{O}\right)$ se halla a $24 \mathrm{~km}$ del límite sudoeste del parque nacional Los Alerces. Su población es de 9908 habitantes. Ambas localidades se ubican en la zona de ecotono bosque-estepa andino patagónicos de Chubut. La principal actividad económica de estas ciudades es la turística. Todos estos enclaves urbanos poseen características multiculturales cuya población proviene de otras áreas urbanas del país, de áreas rurales, son descendientes de inmigrantes y/o de poblaciones originarias.

\section{Muestreo y Análisis de datos}

En cada localidad se seleccionaron al azar cuadras, y en cada una de ellas se registró si la casa presentaba o no plantas leñosas en el cerco. Se trabajó hasta llegar a contabilizar 100 viviendas con cercos vivos de especies leñosas en todas las localidades, con la excepción de San Carlos de Bariloche en que se muestrearon 300 viviendas con cercos vivos dado la mayor cantidad de habitantes. El muestreo se realizó abarcando varios barrios por localidad, excluyendo los barrios céntricos, donde mayormente hay comercios y pocas viviendas particulares, como así también los barrios o loteos nuevos, donde tanto las viviendas como los cercos y jardines se encuentran en construcción. El muestreo solo se realizó sobre los cercos ubicados al frente de las viviendas. Según la ubicación de los lotes o las diferentes urbanizaciones, los frentes presentaban una longitud variable de $12-22 \mathrm{~m}$. El muestreo se realizó desde mediados de septiembre de 2010 a mediados de enero de 2011 a fin de encontrar la mayor cantidad de especies con estructuras reproductivas (flores o frutos) que faciliten $\mathrm{su}$ identificación. En cada vivienda se realizó un inventario de las especies presentes y se colectaron ramas de las especies confeccionándose un herbario de referencia que fue depositado en el Laboratorio Ecotono (UNCo). La filiación taxonómica siguió a Zuloaga et al. (2009).

La sumatoria de las especies encontradas para las ocho ciudades fue considerada la riqueza total de especies en los cercos patagónicos. Por otra parte, la frecuencia de ocurrencia de las especies (Tabla 1) se calculó mediante el número de veces que aparece la especie "ni" en relación al total de cercos analizados nt=1000; se lo considera el índice de consenso más apropiado para estimar la importancia cultural de las especies (Ladio \& Lozada 2004). Las especies fueron clasificadas según su forma de vida en árboles, arbustos, hierbas, enredaderas y lianas según Zuloaga et al. (2009). Asimismo fueron categorizadas según su origen biogeográfico en nativas o exóticas, y en relación a la región biogeográfica de origen (Holártica, Neotropical, Paleotropical, Capense, Australiana y/o Antártica) (Diels 1908, citado en Hueck 1978). Por otra parte, se realizó una exhaustiva revisión bibliográfica de las 30 especies con mayor consenso cultural sobre sus características distintivas, sus factores dimensionales y estéticos (Tabla 1). La revisión bibliográfica fue realizada en las siguientes bases de datos: Scielo (www.scielo.org), Scopus (www.scopus.com) y Scirus (www.scirus.org); se utilizó el nombre de las especies como palabra clave. Además, se realizó una búsqueda sistemática de tesis, libros y capítulos de libros. La riqueza de especies nativas y exóticas fue comparada usando un test binomial $(P<0.05)$.

\section{RESULTADOS}

Los cercos vivos se caracterizaron por ser unidades bastante homogéneas, aunque presentaron asociaciones de especies particulares coexistentes con cercos no vivos de distintos materiales (madera, alambre, hierros, etc.). Su disposición horizontal es en fila siguiendo los límites del frente, en general con una altura de 1 a $3 \mathrm{~m}$. La disposición vertical es principalmente monoestratificada a multiestratificada. De un total de 3058 registros, la riqueza total correspondió a 137 especies diferentes en toda la región considerada. La mayoría de las plantas se determinaron a nivel de especie, salvo para aquellas del género Rosa distintas de Rosa rubiginosa L. y las del género Rhododendron. En su mayoría fueron ornamentales perennes o semiperennes, con follaje denso y/o espinoso. Las flores amarillas y/o rojas, así como los 
Tabla 1. Especies leñosas más utilizadas en los cercos vivos. Se indica el nombre científico, nombre común, familia botánica, características estéticas y frecuencia de uso (\%). En negritas se indican las especies nativas, los superíndices indican si es invasora o adventicia en Argentina.

Table 1. Most used woody species for hedging (scientific and common name, botanical family, aesthetic characteristics and frequency of use, \%). Native species are indicated in bold and superscript indicates author mentions if invasive or adventitia in Argentina.

\begin{tabular}{|c|c|c|c|c|}
\hline Nombre científico & Nombre común & Familia & Características estéticas de las especies & $\begin{array}{l}\text { Frec. de } \\
\text { uso }(\%)\end{array}$ \\
\hline $\begin{array}{l}\text { Cytisus scoparius (L.) } \\
\text { Link }^{12}\end{array}$ & retama & Fabaceae & $\begin{array}{l}\text { Caducifolia, barrera colorida (flores } \\
\text { amarillas) y frondosa. }\end{array}$ & 8.5 \\
\hline Rosa sp & & Rosaceae & Perenne, barrera colorida y espinosa. & 8.0 \\
\hline $\begin{array}{l}\text { Pseudotsuga menziesii } \\
\text { (Mirb.) Franco }\end{array}$ & $\begin{array}{l}\text { pino Oregón, abeto } \\
\text { de Douglas }\end{array}$ & Pinaceae & Conífera perenne, barrera frondosa. & 6.6 \\
\hline $\begin{array}{l}\text { Cupressus macrocarpa } \\
\text { Hartw. ex Gordon }\end{array}$ & $\begin{array}{l}\text { ciprés de Monterrey, } \\
\text { ciprés de California }\end{array}$ & Cupressaceae & Perenne, barrera frondosa. & 4.9 \\
\hline Ligustrum sinense Lour. ${ }^{2}$ & ligustrina & Oleaceae & Perenne o semiperenne, barrera frondosa. & 3.3 \\
\hline Rosa rubiginosa $\mathrm{L}^{1,2}$ & rosa mosqueta & Rosaceae & $\begin{array}{l}\text { Caduca, barrera espinosa, colorida } \\
\text { (flores rosas, frutos rojos) y con frutos } \\
\text { comestibles. }\end{array}$ & 2.9 \\
\hline $\begin{array}{l}\text { Pyracantha coccinea } \mathrm{M} . \\
\text { Roem. }\end{array}$ & $\begin{array}{l}\text { crataegus, espino } \\
\text { de coral, espino de } \\
\text { fuego }\end{array}$ & Rosaceae & $\begin{array}{l}\text { Perenne, barrera espinosa y colorida } \\
\text { (frutos brillantes rojos o anaranjados). }\end{array}$ & 2.7 \\
\hline $\begin{array}{l}\text { Maytenus boaria } \\
\text { Molina }\end{array}$ & maitén & Celastraceae & $\begin{array}{l}\text { Perenne, barrera semifrondosa, hojas y } \\
\text { frutos comestibles. }\end{array}$ & 2.6 \\
\hline Syringa vulgaris L. ${ }^{2}$ & lila, lila común & Oleaceae & $\begin{array}{l}\text { Caduca, barrera semifrondosa, colorida } \\
\text { (grandes y densas inflorescencias lilas u } \\
\text { otros colores) y perfumada. }\end{array}$ & 2.6 \\
\hline Hedera helix L. 2,5,6 & $\begin{array}{l}\text { hiedra, hiedra } \\
\text { trepador }\end{array}$ & Araliaceae & $\begin{array}{l}\text { Enredadera perenne, barrera } \\
\text { semifrondosa, de follaje vistoso. }\end{array}$ & 2.2 \\
\hline $\begin{array}{l}\text { Cotoneaster franchetii } \\
\text { Bois. } .^{1,2}\end{array}$ & & Rosaceae & $\begin{array}{l}\text { Perenne, barrera frondosa, colorida } \\
\text { (frutos rojos o anaranjados brillantes). }\end{array}$ & 1.9 \\
\hline $\begin{array}{l}\text { Aristotelia chilensis } \\
\text { (Molina) Stuntz }\end{array}$ & maqui & Elaeocarpaceae & $\begin{array}{l}\text { Perenne, barrera semifrondosa brillante, } \\
\text { frutos comestibles. }\end{array}$ & 1.8 \\
\hline Prunus laurocerassus L. $^{2}$ & $\begin{array}{l}\text { laurel cerezo, } \\
\text { laurocerezo }\end{array}$ & Rosaceae & $\begin{array}{l}\text { Perenne, } \\
\text { barrera frondosa y vistosa (hojas grandes, } \\
\text { verdes oscuras brillantes). }\end{array}$ & 1.8 \\
\hline Rubus ulmifolius Schott ${ }^{1,2}$ & zarzamora, mora & Rosaceae & $\begin{array}{l}\text { Perenne, barrera semi frondosa, espinosa, } \\
\text { con frutos comestibles. }\end{array}$ & 1.8 \\
\hline Sambucus nigra $\mathrm{L}^{2}$ & sauco & Adoxaceae & $\begin{array}{l}\text { Caduca, barrera semifrondosa, frutos } \\
\text { comestibles. }\end{array}$ & 1.6 \\
\hline Sorbus aucuparia L. ${ }^{1,2}$ & serbal del cazador & Rosaceae & $\begin{array}{l}\text { Caduca, barrera semifrondosa, colorida } \\
\text { (frutos rojos o anaranjados). }\end{array}$ & 1.6 \\
\hline Acer pseudoplatanus L. ${ }^{1,2}$ & $\begin{array}{l}\text { arce blanco, falso } \\
\text { plátano }\end{array}$ & Aceraceae & Caduca, barrera frondosa. & 1.6 \\
\hline $\begin{array}{l}\text { Cupressus arizonica } \\
\text { Greene }\end{array}$ & ciprés de Arizona & Cupressaceae & Conífera perenne, barrera frondosa. & 1.5 \\
\hline $\begin{array}{l}\text { Chusquea culeou E. } \\
\text { Desv. }\end{array}$ & colihue & Poaceae & $\begin{array}{l}\text { Perenne, barrera semifrondosa, cogollos } \\
\text { comestibles. }\end{array}$ & 1.5 \\
\hline $\begin{array}{l}\text { Crataegus monogyna } \\
\text { Jacq. } .^{1,2}\end{array}$ & $\begin{array}{l}\text { cerezo de pastor, } \\
\text { espino albar }\end{array}$ & Rosaceae & $\begin{array}{l}\text { Caduca, barrera colorida (vistosa floración } \\
\text { blanca/roja, vistosa fructificación roja), } \\
\text { semifrondosa. }\end{array}$ & 1.5 \\
\hline $\begin{array}{l}\text { Cotoneaster microphylla } \\
\text { Wall. Ex. Lindl. }\end{array}$ & -- & Rosaceae & $\begin{array}{l}\text { Perenne, berrera colorida (hojas verde } \\
\text { coriáceo, vistosa fructificación roja), } \\
\text { semifrondosa. }\end{array}$ & 1.5 \\
\hline $\begin{array}{l}\text { Lomatia hirsuta (Lam.) } \\
\text { Diels }\end{array}$ & radal & Proteaceae & $\begin{array}{l}\text { Perenne, barrera colorida (hojas verde } \\
\text { brillante), frondosa, uso medicinal. }\end{array}$ & 1.3 \\
\hline Photinia serrulata Lindl. & fotinia, acerolo chino & Rosaceae & $\begin{array}{l}\text { Perenne, barrera frondosa, colorida (hojas } \\
\text { verdes oscuras, brotes nuevos rojizos). }\end{array}$ & 1.2 \\
\hline Prunus cerasifera Ehrh. ${ }^{2}$ & ciruelo silvestre & Rosaceae & $\begin{array}{l}\text { Caduca, barrera colorida (variedades de } \\
\text { hojas purpúreas, densa floración), frutos } \\
\text { comestibles. }\end{array}$ & 1.2 \\
\hline $\begin{array}{l}\text { Nothofagus antarctica } \\
\text { (G. Forst.) Oerst. }\end{array}$ & Nire, roble & Nothofagaceae & $\begin{array}{l}\text { Caduca, barrera semifrondosa, colorida } \\
\text { (en otoño). }\end{array}$ & 1.2 \\
\hline $\begin{array}{l}\text { Nothofagus dombeyi } \\
\text { (Mirb.) Oerst. }\end{array}$ & coihue, coigue & Nothofagaceae & $\begin{array}{l}\text { Perenne, barrera semifrondosa, verde } \\
\text { oscura }\end{array}$ & 1.1 \\
\hline
\end{tabular}


Tabla 1. Continuación.

Table 1. Continuation.

\begin{tabular}{llllc}
\hline Nombre científico & Nombre común & Familia & Características estéticas de las especies & $\begin{array}{l}\text { Frec. de } \\
\text { uso }(\%)\end{array}$ \\
\hline $\begin{array}{l}\text { Cotoneaster dielsiana } \\
\text { Pritz. }\end{array}$ & -- & Rosaceae & $\begin{array}{l}\text { Semiperenne, barrera frondosa, colorida } \\
\text { (frutos rojos o anaranjados brillantes). }\end{array}$ & 1.1 \\
$\begin{array}{l}\text { Malus domestica } \\
\text { Borkh. }{ }^{1,2}\end{array}$ & manzano & Rosaceae & $\begin{array}{l}\text { Caduca, barrera semifrondosa, frutos } \\
\text { comestibles. }\end{array}$ & 1.1 \\
$\begin{array}{l}\text { Schinus patagonicus } \\
\text { (Phil.) I.M. Johnst. ex } \\
\text { Cabrera }\end{array}$ & laura, muchi & Anacardiaceae & $\begin{array}{l}\text { Perenne, barrera } \\
\text { semifrondosa verde oscura, frutos } \\
\text { comestibles. }\end{array}$ & 1.1 \\
\hline
\end{tabular}

${ }^{1}$ Fernández et al. 2007; ${ }^{2}$ Zuloaga et al. 2009; ${ }^{3}$ Simberloff et al. 2002; ${ }^{4}$ Hurrell \& Bazzano 2003; 5 Jankowski et al. 2000;

${ }^{6}$ Heather et al. 2011.

frutos carnosos predominaron en los cercos (Tabla 1); muchos de ellos eran usados como comestible (Rapoport et al. 1999; 2003). El número de especies en promedio por casa fue $3.0 \pm 0.5$.

Las especies con mayor consenso de uso a nivel regional fueron: Cytisus scoparius (L.) Link, Rosa sp., Pseudotsuga menziesii (Mirb.) Franco, Cupressus macrocarpa Hartw. ex Gordon y Ligustrum sinense Lour (Tabla 1). Las especies, reunidas en 48 familias botánicas presentaron un orden decreciente de frecuencia Rosaceae, Fabaceae, Cupressaceae, Pinaceae y Oleaceae, incluyendo en conjunto al $67 \%$ del total de registros (Tabla 1). Las especies fueron principalmente arbustos $(56 \%)$, árboles ( $40 \%$ ) y en menor medida lianas o enredaderas $(4 \%)$.

Del total de especies registradas, la mayoría fueron de origen exótico (105 especies, 76.6\%), entre ellas Cytissus scoparius, Pseudotsuga menziesii, Rosa spp., Cupressus macrocarpa, Ligustrum sinense y Rosa rubiginosa L. (test binomial, $P<0.05$ ), y $23.4 \%$ (32 especies) fueron de origen nativo, entre ellas Maytenus boaria Molina, Aristotelia chilensis (Molina) Stuntz, Chusquea culeou E. Desv., Diostea juncea (Gillies ex Hook.) Miers, Discaria chacaye (G. Don) Tortosa y Embothrium coccineum J.R. Forst. et G. Forst. A nivel regional las especies de los cercos provienen de la región Holártica $(71.5 \%$ del total de especies), de la región Antártica $(23.0 \%)$, Paleotropical $(19.0 \%)$, Neotropical (5.1\%) y Australiana (2.0\%).

Según la revisión bibliográfica, las especies exóticas potencialmenteinvasoras utilizadasen los cercos nor-patagónicos son principalmente Hedera helix L., Pseudotsuga menziesii, Acer pseudoplatanus L., Crataegus monogyna, Sorbus aucuparia (Tabla 1), así como también Ulex europaeus, aunque ésta no se encuentra entre las 30 especies más utilizadas.

\section{DISCUSIÓN}

Este trabajo exploratorio da cuenta del conjunto significativo de especies que son seleccionadas por los habitantes de ciudades patagónicas para la construcción de cercos vivos. Hasta el momento estos espacios han recibido escasa atención. En los relevamientos y en la planificación de las ciudades se han priorizado a las especies que crecen en calles, parques, plazas y jardines (Molina 2009; Puntieri \& Grosfeld 2009), analizando las principales comunidades vegetales en ejidos municipales (Dzendoletas et al. 2006), o las modificaciones de la vegetación por acción del hombre (Dezzotti et al. 2004). La construcción de los cercos es una necesidad para las personas que implica la resolución de problemas ligados a la protección y al límite de la vivienda por lo que la selección de sus especies no es al azar sino que depende de factores tanto socioculturales como biológicoambientales.

Nuestros resultados demuestran y apoyan la hipótesis general que postula que la trayectoria histórica de las ciudades patagónicas estudiadas parece haber influenciado hacia el cultivo de especies de origen exótico y de la región Holártica. Por ejemplo, en el siglo pasado, la estación forestal de la Isla Victoria (dependiente del parque Nahuel Huapi) contaba con un vivero con más de 25 variedades de rosales y más de 100 especies y variedades de distintas Pinaceae y Cupressaceae exóticas que fueron destinadas a enriquecer el arbolado público y privado de la ciudad de Bariloche (Vallmitjana, sin fecha). Esta tendencia, que inclusive fue paradójicamente impulsada por un organismo de conservación, ha sido muy expandida. Este patrón de uso orientado hacia especies exóticas de importancia global e impulsado por los patrones estéticos de colonias de países 
europeos, ha sido evidenciado en estudios de otras regiones del mundo (Dos Santos et al. 2010; Nagendra \& Gopal 2011; Linhares de Souza et al. 2012).

En términos de familias botánicas, este patrón coincide con las familias de mayor importancia global en la horticultura de áreas templadas, siendo Asteraceae, Fabaceae, Chenopodiaceae, Solanaceae y Brassicaceae las más representativas de las floras urbanas actuales (Méndez 2005). Esta preferencia también se evidencia en las plantas que se comercializan en las ciudades mencionadas en viveros y floristerías, por lo que -en la actualidad- la oferta de especies está muy influenciada por este patrón socio-cultural. Con respecto a la forma de vida, los arbustos y árboles son los que ofrecen una matriz más frondosa para ser usada como límite, siendo esta selección coincidente con estudios de cercos realizados en México (Avendaño Reyes \& Acosta Rosado 1999), en Brasil (Nascimento et al. 2009) y en regiones del trópico y subtrópico del mundo (Ospina-Ante 2003). Las características biofísicas son importantes criterios de selección de plantas leñosas, así como también su crecimiento rápido, adaptabilidad y resistencia a la sequía (Ayuk 1997; Cardoso \& Ladio 2011), por lo que es esperable que éstas características biológicasecológicas también influyan en el patrón de selección.

En este trabajo, la presencia de especies nativas (32 especies) indica que los cercos pueden ser considerados unidades de conservación de pequeña escala que están al cuidado de los habitantes urbanos, pero que la inclusión de una mayor riqueza de especies debe ser estimulada. Estudios realizados en Brasil señalan que sus cercos están compuestos por $67 \%$ de nativas (Nascimento et al. 2009), mientras que en México por 91\% (Avendaño Reyes \& Acosta Rosado 1999). Esto sugiere que los cercos patagónicos podrían tener un potencial mayor como una unidad de conservación. Los bosques templados de Argentina y Chile albergan muchas especies nativas atractivas desde un punto de vista ornamental y que de hecho son utilizadas con estos fines en otras partes del mundo (Puntieri \& Grosfeld 2009). Varios autores (Rovere \& Molares 2012) han señalado que sería deseable la utilización de especies nativas para estos fines, a lo cual se sumaría una disminución de los costos y tiempo para su mantenimiento, dado que estas se encuentran adaptadas a las condiciones ambientales de la región.

Sin embargo, muchas de las especies exóticas utilizadas en la composición de los cercos y documentadas en este trabajo ya han sido registradas como invasoras en áreas protegidas aledañas, o son especies potencialmente invasoras (Tabla 1). Entre ellas, Hedera helix se asilvestró en otros lugares de Argentina (Jankowski et al. 2000) y se están desarrollando trabajos de restauración ecológica para su control en bosques templados (Heather et al. 2011). Otro caso es Ulex europaeus, una especie incluida en la lista de las 100 especies más invasoras y agresivas de todo el mundo (Global Invasive Species Database 2012); en el parque Lanín se están restaurando áreas naturales controlando dicha especie (Pérez, Parques Nacionales, comunicación personal). Del mismo modo, Pseudotsuga menziesii ha sido detectada como especie invasora dentro del parque Lanín y Nahuel Huapi (Simberloff et al. 2002; APN 2012). Por ello una mayor utilización de especies nativas permitiría, por un lado, disminuir la dispersión de especies potencialmente invasoras hacia las áreas de interés para la conservación, la formación de corredores biológicos entre las áreas urbanas y las zonas protegidas, como así también ofrecer paisajes florales urbanos en sintonía con su entorno natural más próximo.

\section{CONCLUSIONES}

El rol de los paisajes humanizados como unidades de conservación y restauración ha sido considerado escasamente en la literatura ecológica (Capparrelli et al. 2011). En este sentido, los cercos vivos construidos a la medida y a la manera de las decisiones de las personas, son ámbitos en los cuales los habitantes pueden dar lugar a especies nativas, y de esta manera fomentar su conservación (al tolerarlas y dejarlas en pie en su terrero), o realizar acciones de restauración al cultivar los propágulos en sus predios. Estas acciones, dependen de los valores y la cosmovisión de los individuos, aspectos que no hemos indagado de manera directa en este estudio, pero que completaremos en un futuro con el fin de profundizar, junto con los propietarios, en el tema.

Sin embargo, nuestro relevamiento da cuenta de manera indirecta que más de 30 especies nativas, es decir cerca de $4 \%$ del total de plantas 
nativas que contiene el parque Nahuel Huapi (Ezcurra \& Brion 2005), fueron seleccionadas para ser parte de estos espacios, más otras 100 especies exóticas que dan estructura vegetal horizontal y vertical a los cercos y cumplen funciones ecosistémicas de relevancia. Según varios autores (Harvey et al. 2005; Zahawi 2005) los cercos ofrecen una red interconectada de cobertura vegetal, que brinda una arquitectura vertical especialmente propicia para ser usada como perchas por aves dispersoras, además de proveer sombra que favorece el establecimiento de nuevos propágulos. Por lo tanto, la difusión a la población sobre el uso de especies nativas en los cercos, como Maytenus boaria, Aristotelia chilensis, Chusquea culeou, Diostea juncea, Discaria chacaye y Embothrium coccineum, debería ser estimulada por los organismos nacionales y provinciales, y acompañada por un mayor impulso de su comercialización en los viveros locales.

Por otra parte, también estos organismos debieran alertar a la población sobre los riesgos del uso de ciertas especies invasoras exóticas, como Cytisus scoparius, Rosa rubiginosa, Pseudotsuga menziesii, Ulex europaeus, entre otras que pueden causar perjuicios ambientales y ecológicos graves en los bosques andinopatagónicos. Concluimos quelos conocimientos y valores de las personas y la puesta en práctica de los mismos son componentes cruciales en la interrelación del ser humano y la naturaleza que deben ser incorporados a las propuestas ligadas a la conservación de biodiversidad. La prevalencia de ideales estéticos vinculados a la influencia de los colonos europeos parece haber primado en la selección de las especies exóticas en la región. Sin embargo, estos valores no son estáticos y son flexibles, de modo que es posible crear una mayor conciencia sobre el uso de plantas nativas y que los pobladores urbanos empiecen a recrear una nueva imagen de su entorno urbano.

Agradecimientos: Especial agradecimiento al Consejo Nacional de Investigaciones Científicas y Técnicas (CONICET) y a la Universidad Nacional del Comahue.A Diana Orlov y Ana Girscht por la colaboración en los muestreos e identificación de especies. Este trabajo ha sido financiado por el proyecto PIP 11420100100258 cuyo titular es A. Rovere.

\section{BibLIOGRAFíA}

Álvarez, M; A Arach; P Arias; MR Contreras; IB Damau; et AL. 2009. Árboles de San Martín de los Andes. Guía de reconocimiento. Asociación Civil Pro-Patagonia. San Martín de los Andes, Argentina. Pp. 162.

APN. 2012. Administración de Parques Nacionales.
Sistema de Información de Biodiversidad. Disponible en: www.sib.gov.ar. Consultado el 7 de agosto de 2012.

Avedaño Reyes, S \& I Acosta Rosado. 1999. Plantas utilizadas como cercas vivas en el estado de Veracruz. Madera Bosques, 6:55-71.

AYUK, ET. 1997. Adoption of agroforestry technology: the case of live hedges in the central plateau of Burkina Faso. Agric. Syst., 54:189-206.

BANDIERI, S. 2005. Historia de la Patagonia. Sudamericana S. A. Buenos Aires, Argentina.

Capparelli, A; N Hilgert; A Ladio; V Lema; C Llano; et al. 2011. Paisajes culturales de Argentina: pasado y presente desde las perspectivas etnobotánica y paleoetnobotánica. Revista En Línea de la Asociación Argentina de Ecología del Paisaje, 2:67-79.

Cardoso, MB \& AH Ladio. 2011. Forestación peridoméstica en Patagonia y conocimiento ecológico tradicional: un estudio de caso. Sitientibus Sér. Ciênc. Biol., 11:321-327.

Dezzotti, A; L Sancholuz \& M Naumann. 2004. Cambios vegetacionales antropogénicos en el Cerro Catedral (Río Negro, Argentina). Bosque, 25:63-71.

Dos Santos, AR; CF Da Rocha \& HG Bergallo. 2010. Native and exotic species in the urban landscape of the city of Rio di Janeiro, Brazil: density, richness and arboreal deficit. Urban Ecosyst., 13:209-222.

Dugue-Brasil, R; GT Soldati; MM EsPíRITO-SANTO; MQ Rezende; S DÁngelo-Neto; et Al. 2011. Composição, uso e conservação de espécies arbóreas em quintais de agricultores familiares na região da mata seca nortemineira, Brasil. Sitientibus Sér. Ciênc. Biol., 11:287-297.

Dzendoletas, MA; S Cavallaro; E Crivelli \& F Pereyra. 2006. Mapa de vegetación del ejido municipal de San Carlos de Bariloche y alrededores, Río Negro Patagonia Argentina. Ecología, 20:65-88.

Eyssartier, C; AH Ladio \& M Lozada. 2011. Traditional horticultural knowledge change in a rural population of the Patagonian steppe. J. Arid Environ., 75:78-86.

Ezcurra, C \& C Brion. 2005. Plantas del Nahuel Huapi. Catálogo de la Flora Vascular del Parque Nacional Nahuel Huapi, Argentina. Universidad Nacional del Comahue, Red Latinoamericana de Botánica. Bariloche, Argentina. Pp. 70.

FAGGI, AM \& J DADON. 2010. Vegetation changes associated to coastal tourist urbanizations. Multequina, 19:53-76.

Fernández, N; M Mermoz \& J Puntieri. 2007. Plantas exóticas invasoras en los Parques Nacionales de Patagonia. Delegación Regional Patagonia, Administración de Parques Nacionales. Bariloche, Argentina. Pp. 46.

Global Invasive Species Database. 2012. Disponible en: www.issg.org/database/welcome/. Consultado el 1 de Octubre de 2012.

Haene, E \& G Aparicio. 2007. 100 Árboles Argentinos. Editorial Albatros. Buenos Aires, Argentina. Pp. 128.

Harvey, CA; C Villanueva; J Villacis; M Chacón; D MUÑOZ; ET AL. 2005. Contribution of live fences to the ecological integrity of agricultural landscapes. Agr. Ecosyst. Environ., 111:200-230.

Heather, EL; M Huso; KC Stanley \& T Taylor. 2011. Composting one invasive species to control another. Restor. Ecol., 19:1-4.

Hueck, K. 1978. Los bosques de Sudamérica: ecología, composición e importancia económica. Sociedad alemana de cooperación técnica Ltda. Eschborn. República Federal de Alemania. Pp. 476. 
Hurrel, JA \& DH Bazzano. 2003. Arbustos 1. Nativos y exóticos. Biota Rioplatense Vol. VIII. Editorial L.O.L.A. Buenos Aires, Argentina. Pp. 263.

Jankowski, L; D Bazzano, A SÁEnz; M Tourn \& G RoITMAN. 2000. Plantas trepadoras nativas y exóticas. Biota Rioplatense Vol. V. Editorial L.O.L.A. Buenos Aires, Argentina. Pp. 264.

LADIO, AH \& M DAMASCOS. 2000. La invasión de plantas exóticas y la conservación de plantas nativas en baldíos suburbanos. Medio Ambiente, 13:27-34.

LAdio, AH \& M Lozada. 2004. Patterns of use and knowledge of wild edible plants in distinct ecological environments: A case study of a Mapuche community from Northwestern Patagonia. Biodiv. Conserv., 13:11531173.

Ladio, A \& S Molares. 2010. Aspectos do estudo da dinâmica do uso de produtos etnobiológicos não tradicionais. Pp. 367-378 en: Albuquerque, UP; RF Paiva de Lucena \& LVFC Cunha (eds.). Métodos e Técnicas na Pesquisa Etnobiológica e Etnoecológica. NUPEEA, Recife, Brazil.

Linhares de Souza, A; R Ferreira; A De Mello; D Da Rocha Plácido; C Zoaid Alves Dos Santos; et AL. 2012. Diagnóstico quantitativo e qualitativo da arborização das praças de aracaju, SE. Rev. Árvore, 35:1253-1263.

MCKINNEY, ML. 2006 Urbanization as a major cause of biotic homogenization. Biol. Conserv., 127:247-260.

MÉNDEZ, E. 2005. Flora y vegetación del centro urbano de Luján de Cuyo. Mendoza (Argentina). Rev. FCA UNCuyo, 1:67-74.

Mermoz, M; C Úbeda; D Grigera; C Brion; C Martin; et AL. 2009. El Parque Nacional Nahuel Huapi. Sus características ecológicas y su estado de conservación. Administración de Parques Nacionales. Bariloche, Argentina. Pp. 80.

Molina, A. 2009. Arbolado Urbano de la ciudad de Esquel, propuesta para su ordenación. Tesis de Ingeniería Forestal, Universidad San juan Bosco. Esquel, Argentina.

NAGENDRA, H \& D GOPAL. 2011. Tree diversity, distribution, history and change in urban parks: studies in Bangalore, India. Urban Ecosyst., 14:211-223.

Nascimento, VT; LG Sousa; GC Alves; EL Araujo \& UP AlbuQUeRQUe. 2009. Rural fences in agricultural landscape and their conservation role in an area of caatinga (dryland vegetation) in Northeast Brazil. Environ. Dev. Sustain., 11:1005-1029.

Ospina-Ante, A. 2003. Agroforestería. Aportes Conceptuales, metodológicos y Prácticos para el Estudio Agroforestal. Asociación delColectivo de Agroecología delSurroccidente Colombiano. Santiago de Cali. Colombia. Pp. 209.

Parra, F; JJ Blancas \& A Casas. 2012. Landscape management and domestication of Stenocereus pruinosus (Cactaceae) in the Tehuacán Valley: human guided selection and gene flow. J. Ethnobiol. Ethnomed., 8:32.

Puntieri, JG \& JE Grosfeld. 2009. Arbolado urbano en la Patagonia andina: buscando el equilibrio. Desde la Patagonia: difundiendo saberes, 9:2-9.

RAPOPORT, EH. 1988. Lo Bueno y lo malo tras el Descubrimiento de América. El punto de vista ecológico y biogeográfico. Arbor, 131:103-125.

RAPOPORT, EH; AH LAdIO \& E SANZ. 1999. Plantas Nativas
Comestibles de la Patagonia andina argentino-chilena. Parte I. Ediciones Alternatura. National Geographic Society. Bariloche, Argentina. Pp. 81.

RApoport, EH; AH LAdio \& E SANZ. 2003. Plantas Nativas Comestibles de la Patagonia andina argentino-chilena. Parte II. Ediciones Alternatura. Secretaría de Extensión Universitaria. UNC. Fundación Antorchas. Bariloche, Argentina. Pp. 79.

RODRíGUEZ-LAREDO, D. 2008. El valor ecológico, ornamental $y$ de uso tradicional de las especies nativas de la ciudad de La Paz. Universidad de San Andrés. Facultad de Arquitectura, Artes, Diseño y Urbanismo. Instituto de Investigaciones y Postgrado. La Paz, Bolivia.

Rovere, AE \& S Molares. 2012. Una estrategia interjurisdiccional para el control de especies ornamentales invasoras. Libro de Actas de Eco Reuniones. Eco-sociedad 2012, 256-263.

RudD, H; J VAla \& V Schaefer. 2002. Importance of Backyard Habitat in a Comprehensive Biodiversity Conservation Strategy: A Connectivity Analysis of Urban Green Spaces. Restor. Ecol., 10:368-375.

SARASOlA, MM; VE Rusch; TM SCHLICHTER \& CM GHERSA. 2006. Invasión de coníferas forestales en áreas de estepa y bosque de ciprés de la cordillera en la región Andino Patagónica. Ecol. Austral, 16:143-156.

Sedrez Dos Reis, M \& A Ladio. 2012. Nuevos enfoques para la conservación desde la perspectiva del paisaje cultural: las Araucarias Sudamericanas. Libro de Actas de Eco Reuniones. Eco-Sociedad 2012, 117-130.

Simberloff, D; MA Relva \& M Nuñez. 2002. Gringos en el Bosque: Introduced tree invasion in a Nothofagus/ Austrocedrus forest. Biol. Invas., 4:35-53.

Siviero, A; TD Delunardo; M Haverroth; LC De Oliveira \& AM Silva Mendonça. 2011. Cultivo de Espécies Alimentares em Quintais Urbanos de Rio Branco, Acre, Brasil. Acta Bot. Brasílica, 25:549-556.

Teruya Eichemberg, M; MC De Mello Amorozo \& L Cunha DE MourA. 2009. Species composition and plant use in old urban homegardens in Rio Claro, Southeast of Brazil. Acta Bot. Brasilica, 23:1057-1075.

Valverde, S; G Maragliano; M Impemba \& F Trentini. 2011. Procesos históricos, transformaciones sociales $y$ construcciones de frontera. Aproximaciones a las relaciones interétnicas. Estudios sobre Norpatagonia, Argentina y Labrador, Canadá. Editorial de la Facultad de Filosofía y Letras, Universidad de Buenos Aires. Buenos Aires, Argentina.

Vallmitjana, R. (s f). Isla Victoria. Archivo Histórico Regional. S.C. de Bariloche, Argentina.

Vogl, CR; B Vogl-Lukasser \& RK Puri. 2004. Tools and Methods for Data Collection in Ethnobotanical Studies of Homegardens. Field Methods, 16:285-306.

ZAHAWI, RA. 2005. Establishment and growth of living fence species: an overlooked tool for the restoration of degraded areas in the tropics. Restor. Ecol., 13:92-102.

Zuloaga, FO; O Morrone \& MJ Belgrano. 2009. Catálogo de las plantas vasculares del Cono Sur. Disponible en: www.darwinion.gov.ar. Consultado: mayo 2011. 